\title{
Apoio social e qualidade de vida de famílias de crianças com cardiopatia congênita
}

\author{
Social support and quality of life of families with children \\ with congenital heart disease
}

Gisele Vilella da Silva (https://orcid.org/0000-0002-2628-2873) ${ }^{1}$

Denise Ely Bellotto de Moraes (https://orcid.org/0000-0002-3562-0926) ${ }^{1}$

Tulio Konstantyner (https://orcid.org/0000-0002-7931-9692) ${ }^{1}$

Heitor Pons Leite (https://orcid.org/0000-0001-8260-7559) ${ }^{1}$

${ }^{1}$ Departamento de Pediatria, Universidade Federal de São Paulo. R. Botucatu 598, Vila Clementino. 04023-062 São Paulo SP Brasil.

gvilelasil@gmail.com

\begin{abstract}
The scope of this article is to evaluate to what extent social support for families with children with congenital disease impacts their quality of life. It involved a cross-sectional study with 254 parents of children with congenital heart disease. A semi-structured interview was conducted with the parents, using the Ecomap and the quality of life (World Health Organization Quality of Life Bref) and social support (Medical Outcomes Study) scales. Receiving social support was positively correlated with quality of life ( $r=0.535$; $p<0.001)$. A 10-point increase in the social support scale led to a 3-point increase in the total score of the quality of life scale ( $\beta=0.30$; $C 195 \%$ : 0.23 ; 0.37). Socioeconomic conditions were associated with quality of life ( $\beta=0.27$; CI95\%: 0.11; $0.43)$ and caregivers who reported not having leisure activity ( $\beta=-3.27$; $C 195 \%-5.55 ;-1.12)$ and who were undergoing health treatment $(\beta=-2.86$; C195\%: -5.55 ; - 0.17$)$ had a lower perception of quality of life. Negative consequences to the quality of life of caregivers taking care of a child with congenital heart disease can be intensified by a lack of leisure activity and having health problems. Adequate social support and socioeconomic resources positively influence the quality of life of these caregivers.
\end{abstract}

Key words Quality of life, Social support, Congenital abnormalities, Family, Caregivers
Resumo O objetivo deste artigo é avaliar em que medida o apoio social a famílias de crianças com cardiopatia congênita impacta na qualidade de vida. Estudo transversal com 254 pais de crianças com cardiopatia congênita. Foi realizada entrevista semiestruturada com os pais, utilizando o ecomapa e as escalas de qualidade de vida (World Health Organization Quality of Life Bref) e de suporte social (Medical Outcomes Study). O apoio social teve correlação positiva com a qualidade de vida $(r=0,535 ; p<0,001)$. O aumento de 10 pontos na escala de suporte social levou ao aumento de 3 pontos no escore total da escala de qualidade de vida ( $\beta=0,30$; IC95\%: 0,23; 0,37). A condição socioeconômica associou-se positivamente com qualidade de vida ( $\beta=0,27$; IC95\%: 0,$11 ; 0,43)$ e os cuidadores que referiram não ter atividade de lazer $(\beta=-3,27$; IC95\% $-5,55 ;-1,12)$ e que estavam em tratamento de saúde ( $\beta=-2,86$; IC95\%: $-5,55$; $-0,17)$ tiveram menor percepção na qualidade de vida. Consequências negativas à qualidade de vida de cuidadores de crianças com cardiopatia congênita podem ser intensificadas pela falta de atividade de lazer e problemas de saúde. Apoio social e condição socioeconômica adequados são fatores que influenciam positivamente na qualidade de vida destes cuidadores.

Palavras-chave Qualidade de Vida, Apoio Social, Anormalidades Congênitas, Família, Cuidadores 


\section{Introdução}

A cardiopatia congênita é definida como máformação na estrutura e função do coração ${ }^{1}$. De acordo com o DATASUS, a incidência de cardiopatia congênita no Brasil foi de aproximadamente 3,6 para cada 10.000 nascidos vivos no período entre 1999 e $2015^{2}$.

O avanço tecnológico tem contribuído para o diagnóstico precoce e para a melhora no tratamento, o que reduziu significativamente a mortalidade e aumentou o tempo de sobrevida dos pacientes $^{3}$. No entanto, o maior tempo de vida dos pacientes tem acarretado dificuldades de enfrentamento da doença por parte das famílias, que nem sempre apresentam rede de apoio efetiva ${ }^{4}$.

O diagnóstico de cardiopatia congênita causa grande impacto aos pais, em consequência de tratamentos invasivos recorrentes, cirurgia e maior risco de óbito do filho ${ }^{4}$. Desencadeia nos seus cuidadores estresse e maior probabilidade de doenças relacionadas à saúde mental ${ }^{5}$. Tais implicações podem influenciar de modo negativo a qualidade de vida dessas famílias ${ }^{6}$.

Além dos prejuízos à saúde mental, os pais têm menor tempo de lazer e muitas vezes precisam abdicar da profissão para se dedicar ao tratamento de saúde do filho, o que resulta na sobrecarga de trabalho e, consequentemente, no isolamento social e déficit financeiro familiar ${ }^{6,7}$.

Estudos sobre qualidade de vida dos pais sugerem que promover intervenções para ampliar a rede social e o apoio social e fortalecer os vínculos já existentes pode favorecer a identificação precoce de problemas relacionados à saúde mental e abrandar as dificuldades advindas da doença do filho ${ }^{6,8}$. Por outro lado, esta afirmação não é unanime, uma vez que não foi verificada associação entre apoio social e saúde mental de pais de crianças com câncer?.

Nesse contexto, desenvolver estudos que investiguem a associação entre as características de apoio potencialmente fornece informações para elaborar estratégias para melhorar a qualidade de vida dos cuidadores. O objetivo deste estudo foi avaliar em que medida o apoio social afeta a qualidade de vida das famílias de crianças com cardiopatia congênita.

\section{Métodos}

\section{Desenho do estudo e participantes}

Trata-se de estudo transversal, do qual participaram cuidadores de crianças ou adolescentes com cardiopatia congênita do Ambulatório de Cardiologia Pediátrica de um hospital universitário de São Paulo. Foram excluídos cuidadores que tiveram dificuldade de compreender ou responder às questões apresentadas, cuidadores de crianças institucionalizadas, cuidadores que apenas acompanhavam a criança na consulta e pais menores de 18 anos.

Utilizou-se uma amostra de conveniência de pacientes atendidos no período de agosto a dezembro de 2016. Dos 300 pacientes que atendiam os critérios de inclusão, 254 aceitaram participar do estudo e 46 não aceitaram. A amostra foi, portanto, de 254 participantes.

\section{Aspectos éticos}

Após o estudo ser aprovado pelo Comitê de Ética em Pesquisa da Universidade de São Paulo, foi realizada pesquisa de prontuário para identificar potenciais participantes. A coleta foi iniciada posteriormente ao aceite e assinatura do termo de consentimento livre e esclarecido.

\section{Coleta de dados}

Para identificar as variáveis deste estudo foram utilizados na coleta de dados cinco questionários. As características sociodemográficas dos participantes foram coletadas por meio de entrevistas semiestruturadas. As informações sobre dados clínicos dos pacientes foram obtidas por consulta em prontuários.

O ecomapa é um diagrama que expõe a relação entre a família e as redes sociais existentes na comunidade. É um instrumento que ajuda a quantificar e avaliar os apoios disponíveis na rede e sua utilização pela família; além disso, possibilita identificar o fluxo ou a falta de recursos e as privações vivenciadas pela família na sociedade ${ }^{10}$.

Para estimar o nível socioeconômico foram adotados os critérios de Classificação Econômica do Brasil propostos pela Associação Brasileira de Empresas de Pesquisa (ABEP). Esse questionário verifica a posse e utilização de determinados objetos, bens de consumo e o grau de instrução do chefe da família. A condição socioeconômica da família foi estimada de acordo com o total de pontos obtidos $(0 \text { a } 34)^{11}$. 
A qualidade de vida dos cuidadores foi medida por intermédio do instrumento World $\mathrm{He}$ alth Organization Quality of Life - Abbreviated (WHOQOL-BREF), que foi traduzido e validado no Brasil ${ }^{12}$. Trata-se de um instrumento abreviado de avaliação da percepção da qualidade de vida elaborado pela Organização Mundial da Saúde (OMS) ${ }^{13}$.

Para este estudo, o WHOQOL-BREF foi aplicado no formato abreviado, que é composto por 26 questões que avaliam 4 domínios: físico, psicológico, de relações sociais e meio ambiente. Cada questão tem 5 opções de respostas que variam de 1 (muito ruim) a 5 (muito bom); quanto maior a pontuação do escore total, maior é a percepção de qualidade de vida.

E, por fim, o apoio social foi medido pela escala Medical Outcomes Study (MOS), cujo objetivo é avaliar a funcionalidade e as dimensões do apoio $^{14}$. Entre 2001 e 2005, o presente instrumento foi traduzido, adaptado e validado no Brasil $^{15}$. Essa escala é composta por 19 questões cujas opções de resposta variam de 1 (nunca) a 5 (sempre) e possui 3 domínios: material, afetivo/ interação social e emocional/informação.

\section{Análise estatística}

Inicialmente, foram realizadas análises descritivas. As variáveis categóricas foram apresentadas por frequência absoluta e porcentagem, e as variáveis contínuas foram descritas por mediana e intervalo interquartil. Para a análise da normalidade foram utilizados o teste Shapiro-Wilk e histograma de frequência. A correlação entre os escores geral das escalas WHOQOL-BREF e MOS foi avaliada pelo teste de correlação de $P e$ arson.

Optou-se pelo modelo de regressão linear, uma vez que a variável dependente apresentou normalidade e a análise dos resíduos demonstrou distribuição normal e variância constante. No modelo de regressão linear, foi utilizado como variável dependente o escore geral da escala WHOQOL-BREF e, como principal variável independente, o escore geral da escala MOS. Foram consideradas também outras variáveis independentes: dados sociodemográficos dos cuidadores, condição socioeconômica e dados clínicos dos pacientes. As variáveis com valor de $\mathrm{p}<0,20$ na análise univariada foram inseridas no modelo de regressão linear múltiplo. Foram consideradas como significantes no modelo as associações com valor de $\mathrm{p}<0,05$ com ajuste para sexo e idade do paciente. Utilizou-se a técnica Stepwise Backward para a construção do modelo múltiplo e foi testada a interação entre as variáveis que permaneceram no modelo final. Todas as análises estatísticas foram realizadas utilizando a versão 14.0 do pacote estatístico Stata (Stata Statistical Software: Release 14. College Station, TX, EUA).

\section{Resultados}

As principais características dos pacientes e seus cuidadores são mostradas na Tabela 1. A mediana do escore total da escala WHOQOL-BREF foi 88 (intervalo interquartil $=81-94)$. $O$ escore total da escala $M O S$ apresentou mediana 79 (intervalo interquartil $=64-89)$ e a mediana do ecomapa, que quantifica o apoio percebido pelos cuidadores, foi 4 (intervalo interquartil $=3-5$ ).

Houve correlação positiva significante entre os escores das escalas WHOQOL-BREF e MOS $(\mathrm{r}=0,535-p=<0,001)$. Além disso, houve correlação positiva entre os três domínios da escala MOS e o escore total da escala WHOQOL-BREF (Figura 1). Na análise post hoc com tamanho amostral de 254 indivíduos e com erro alfa 0,05, o coeficiente de correlação encontrado resultou em poder do teste maior do que $99 \%$.

A Tabela 2 mostra o resultado das análises de regressão linear simples e múltipla, em que o sexo e a idade do paciente foram considerados como variáveis de ajuste. Observou-se nesse modelo múltiplo que cada aumento de 10 pontos no escore total da MOS corresponde ao aumento de 3 pontos no escore total da escala WHOQOL-BREF. A cada 10 pontos a mais no escore total da escala de classificação socioeconômica ABEP, o escore total de qualidade de vida aumenta 2,7 pontos. Os pais que relataram não ter atividade de lazer têm o escore total da WHOQOL-BREF diminuído em 2,97. Da mesma forma, o escore dos cuidadores que referiram fazer tratamento de saúde decresce 2,86 na escala de qualidade de vida.

Foi feito o teste de interação entre as variáveis que permaneceram no modelo final, e o resultado não foi significante. No modelo múltiplo, o $\mathrm{R}^{2}$ (coeficiente de determinação) foi 0,35 entre as variáveis que permaneceram no modelo final, e a variável apoio social foi a que apresentou maior coeficiente de determinação: $R^{2}=0,29$.

\section{Discussão}

No presente estudo, observamos correlação positiva do escore total da escala de apoio social e 
Tabela 1. Características epidemiológicas das crianças e características sociodemográficas e socioeconômicas de seus cuidadores ( $n=254 / 254)$.

\begin{tabular}{|c|c|c|}
\hline Variáveis & $\mathbf{n}$ & Valores ${ }^{\star}$ \\
\hline \multicolumn{3}{|l|}{ Características crianças } \\
\hline Idade do paciente (meses) & 57 & $22-117^{\mathrm{a}}$ \\
\hline Sexo (masculino) & 128 & $50,4(44,2-56,5)$ \\
\hline \multicolumn{3}{|l|}{ Escolaridade } \\
\hline $\begin{array}{l}\text { Não frequenta creche/ } \\
\text { escola }\end{array}$ & 97 & $38,2(32,4-44,3)$ \\
\hline $\begin{array}{l}\text { Frequenta creche/ } \\
\text { escola }\end{array}$ & 157 & $61,8(55,6-67,6)$ \\
\hline $\begin{array}{l}\text { Tempo de estudo do } \\
\text { paciente (anos) }\end{array}$ & 6 & $3-8^{a}$ \\
\hline Submetidos à cirurgia & 119 & $46,8(40,7-53,0)$ \\
\hline $\begin{array}{l}\text { Tipo de cirurgia } \\
\text { (corretiva) }\end{array}$ & 69 & $58,5(49,3-67,1)$ \\
\hline \multicolumn{3}{|l|}{$\begin{array}{l}\text { Classificação de risco } \\
\text { cirúrgico (RACHS) }\end{array}$} \\
\hline 1 & 11 & $5,0(2,8-8,8)$ \\
\hline 2 & 137 & $62,0(55,4-68,2)$ \\
\hline 3 & 64 & $29,0(23,3-35,3)$ \\
\hline 4 & 9 & $4,1(2,1-7,8)$ \\
\hline Cardiopatia cianogênica $^{c}$ & 80 & $37,2(30,9-43,9)$ \\
\hline \multicolumn{3}{|l|}{ Síndrome associada } \\
\hline $\operatorname{Sim}$ & 47 & $18,5(14,2-23,8)$ \\
\hline Não & 191 & $75,2(69,5-80,1)$ \\
\hline Em investigação & 16 & $6,3(3,9-10,1)$ \\
\hline \multicolumn{3}{|l|}{$\begin{array}{l}\text { Características } \\
\text { cuidadores }\end{array}$} \\
\hline $\begin{array}{l}\text { Idade do participante } \\
\text { (anos) }\end{array}$ & 34 & $28-40^{\mathrm{a}}$ \\
\hline Sexo (feminino) & 231 & $90,9(86,7-93,9)$ \\
\hline Sem companheiro(a) & 65 & $25,6(20,6-31,3)$ \\
\hline Tempo de estudo (anos) & 12 & $09-12^{\mathrm{a}}$ \\
\hline $\begin{array}{l}\text { Não exercem atividade } \\
\text { laboral }\end{array}$ & 133 & $52,4(46,1-58,5)$ \\
\hline $\begin{array}{l}\text { Tratamento de saúde } \\
\text { contínuo }\end{array}$ & 56 & $22,0(17,3-27,6)$ \\
\hline Sem atividade de lazer & 144 & $56,7(50,5-62,7)$ \\
\hline ABEP & 23,5 & $18-29^{\mathrm{a}}$ \\
\hline \multicolumn{3}{|c|}{$\begin{array}{l}{ }^{*} \text { Valores expressos em porcentagem e IC95\% = intervalo } \\
\text { de confiança; }{ }^{a} \text { Valores expressos em mediana e intervalo } \\
\text { interquartil; }{ }^{b} \mathrm{n}=221 \text { RACHS: Risk Adjustment in Congenital } \\
\text { Heart Surgery; }{ }^{c} \mathrm{n}=215 \text {; ABEP: Associação Brasileira de } \\
\text { Empresas de Pesquisas. }\end{array}$} \\
\hline
\end{tabular}

seus domínios (material, afetivo/interação social e emocional/informação) com o escore total da escala de qualidade de vida. Além disso, a condição socioeconômica associou-se à percepção positiva da qualidade de vida. Paralelamente, não ter atividade de lazer e fazer tratamento de saúde associaram-se com a percepção negativa de qualidade de vida.

Não encontramos pesquisas na literatura que tenham estudado especificamente a relação entre o apoio social e a qualidade de vida de cuidadores de crianças com doença cardíaca. Alguns estudos mostram que dedicar-se aos cuidados de alguém de forma contínua e prolongada, com pouco ou nenhum apoio social, pode influenciar de forma negativa a qualidade de vida dos cuidadores ${ }^{16}$. Em estudo com cuidadores de idosos dependentes, receber ajuda associou-se a maiores escores do domínio relações sociais da escala WHOQOL -BREF ${ }^{17}$.

De modo similar, a percepção de qualidade de vida por parte de mães de crianças com doenças crônicas foi menor quando comparada à de mães de crianças sem doenças crônicas ${ }^{18}$. Em outros estudos realizados com pais de crianças cardiopatas e pais de crianças com câncer, foram constatados piores resultados em todas as dimensões da escala de qualidade de vida relacionada à saúde quando comparados aos de pais de crianças saudáveis e com doenças mais simples, como infecção do trato respiratório superior e doença diarreica $^{6,9}$.

Embora esses estudos não tenham considerado o apoio social como preditor da qualidade de vida, da forma como fizemos nesta pesquisa, os autores sugerem que o apoio social tem impacto positivo na qualidade de vida das mães ${ }^{6,18}$, o que está em concordância com os nossos resultados. Além disso, o capital social, definido como os benefícios advindos da cooperação entre indivíduos e grupos, do qual resulta o apoio social, parece proteger contra problemas de saúde física, porém não de saúde mental de pais de crianças com câncer ${ }^{9}$.

Entretanto, a maioria das pesquisas que estudam o apoio social utiliza a metodologia qualitativa. Estes estudos mostram que cuidadores de crianças cardiopatas são sobrecarregados por fatores como o tempo de cuidado, instabilidade financeira e problemas relacionados à saúde mental. Dadas as diferenças que possam existir entre os cuidados de crianças com câncer e com cardiopatia congênita, tais fatores podem demandar mais apoio social ao cuidador do que a gravidade da doença do paciente ${ }^{7}$.

Pesquisas qualitativas com famílias de crianças com cardiopatia congênita mostram que "ter com quem contar", seja em grupo, presencialmente ou de forma online, possibilita esclarecer dúvidas e proporcionar melhor comunicação en- 

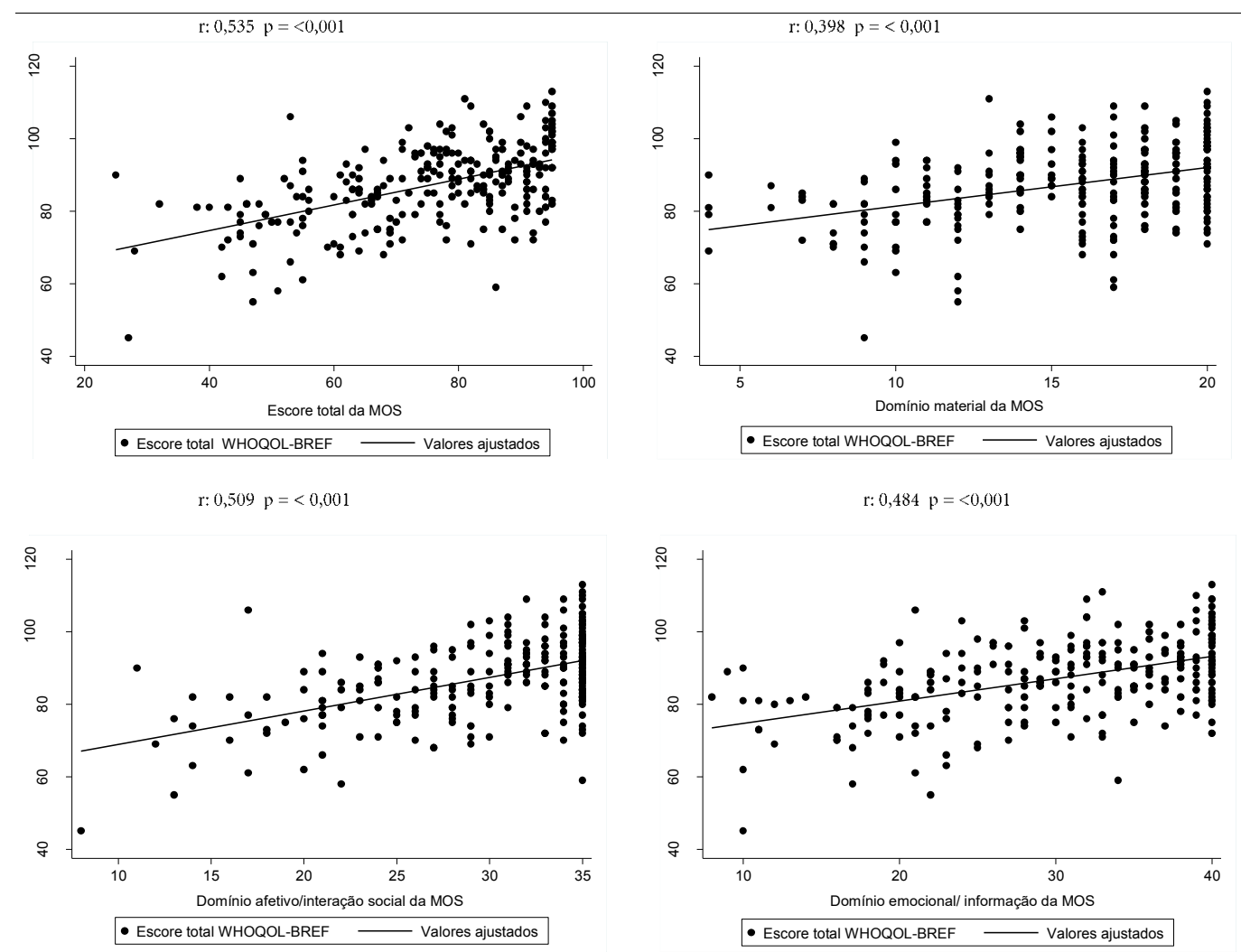

Figura 1. Coeficientes de Correlação de Pearson entre o escore da escala WHOQOL-BREFa e escore total e domínios da escala MOSb (254).

aWHOQOL-BREF: World Health Organization Quality of Life Group-Abbreviated; bMOS: Medical Outcomes Study.

tre paciente, médico e famílias ${ }^{19,20}$, caracterizando o apoio como um fator protetor da qualidade de vida $^{21}$. Entretanto, a necessidade de apoio social dessas famílias é dinâmica, ou seja, pode mudar no decorrer do tratamento e ao longo do desenvolvimento da criança. A necessidade de apoio é maior em momentos mais críticos, tornando-se menor em estágios mais controlados da doença, principalmente quando não há procedimentos invasivos $^{22}$. Para que o apoio social funcione de forma efetiva na vida destas famílias, é necessário que o suporte oferecido seja compatível com as expectativas de quem o recebe; caso contrário, o apoio social será percebido como ausente ou negativo $^{23}$

Adicionalmente, a maior condição socioeconômica dos cuidadores associou-se a uma maior percepção de qualidade de vida. Resultados encontrados em um estudo com mães de crianças com paralisia cerebral corroboram os nossos achados ao constatar que níveis socioeconômi- cos mais elevados estão associados com maior satisfação com a vida ${ }^{24}$. Além disso, outro estudo enfatiza os prejuízos advindos da renda precária dos cuidadores e mostram que famílias com baixa renda têm acesso limitado aos serviços educacionais, sociais e de saúde ${ }^{25}$. Esta dificuldade pode impossibilitar que os pais tenham a chance de usufruir e proporcionar aos filhos mais conforto e bem-estar ${ }^{26}$.

Outro aspecto a ser considerado refere-se às dificuldades inerentes à complexidade do tratamento e aos cuidados exigidos pela doença da criança, que mudam a rotina da família e podem afetar diretamente a vida pessoal e profissional do cuidador. Alguns pais têm dificuldade para manter o emprego, o que restringe seus recursos financeiros, que passam a ser utilizados apenas para as necessidades básicas da família e para o tratamento da criança ${ }^{26}$. Como consequência da redução da renda familiar, há prejuízo da percepção de qualidade de vida por parte das famílias ${ }^{6}$. 
Tabela 2. Análises de regressão linear simples e múltipla* entre as características dos pacientes e seus cuidadores, e o escore total e os domínios da escala de apoio social medido pela $M O S^{\text {a }}$, dados clínicos do paciente e dados sociodemográficos dos cuidadores (254).

\begin{tabular}{|c|c|c|c|c|c|c|c|}
\hline \multirow{3}{*}{ Variáveis } & \multirow{3}{*}{ Categoria de risco } & \multicolumn{3}{|c|}{ Análise univariada } & \multicolumn{3}{|c|}{ Análise multivariada } \\
\hline & & \multicolumn{3}{|c|}{ WHOQOL-BREF $^{\mathrm{b}}$} & \multicolumn{3}{|c|}{ WHOQOL-BREF } \\
\hline & & $\boldsymbol{\beta}$ & IC95\% & P-valor & $\beta$ & IC95\% & P-valor \\
\hline \multicolumn{8}{|l|}{ Pacientes } \\
\hline Idade (meses) & & $-0,01$ & $-0,03 / 0,01$ & 0,358 & - & - & - \\
\hline Sexo & Masculino & $-0,89$ & $-3,57 / 1,78$ & 0,511 & - & - & - \\
\hline Síndrome & & 0,64 & $-2,82 / 4,11$ & 0,715 & - & - & - \\
\hline Submetido à cirurgia & & 0,34 & $-2,34 / 3,02$ & 0,804 & - & - & - \\
\hline $\begin{array}{l}\text { Submetido à cirurgia } \\
\text { paliativa }\end{array}$ & & 1,97 & $-2,19 / 6,14$ & 0,350 & - & - & - \\
\hline RACHS $^{c}$ & $>3$ & $-0,14$ & $-2,44 / 2,17$ & 0,906 & - & - & - \\
\hline Cianogênica $^{\mathrm{d}}$ & Sim & 0,54 & $-2,45 / 3,53$ & 0,722 & - & - & - \\
\hline \multicolumn{8}{|l|}{ Cuidadores } \\
\hline Idade (anos) & & $-0,09$ & $-0,24 / 0,06$ & 0,245 & - & - & - \\
\hline Educação (anos) & & 0,80 & $0,39 / 1,22$ & $<0,001$ & 0,22 & $-0,17 / 0,61$ & 0,268 \\
\hline Estado civil & Sem companheiro & $-0,91$ & $-3,97 /-2,15$ & 0,558 & - & - & - \\
\hline Sem atividade de lazer & & $-4,80$ & $-7,43 /-2,17$ & $<0,001$ & $-2,97$ & $-5,55 /-1,12$ & 0,003 \\
\hline Em tratamento de saúde & & $-2,31$ & $-5,53 / 0,90$ & 0,158 & $-2,86$ & $-5,55 /-0,17$ & 0,037 \\
\hline Sem atividade laboral & & 3,39 & $0,75 / 6,04$ & 0,012 & 0,69 & $-1,53 / 2,92$ & 0,539 \\
\hline $\mathrm{ABEP}$ & & 0,53 & $0,36 / 0,70$ & $<0,001$ & 0,27 & $0,11 / 0,43$ & 0,001 \\
\hline Ecomapa & & 1,48 & $0,77 / 2,19$ & $<0,001$ & 0,57 & $-0,78 / 0,67$ & 0,876 \\
\hline \multicolumn{8}{|l|}{ MOS } \\
\hline Material & & 1,07 & $0,76 / 1,37$ & $<0,001$ & - & - & - \\
\hline Afetivo/Social & & 0,92 & $0,73 / 1,12$ & $<0,001$ & 0,32 & $-0,07 / 0,72$ & 0,110 \\
\hline Emocional/Informação & & 0,62 & $0,48 / 0,75$ & $<0,001$ & 0,36 & $-0,43 / 0,50$ & 0,879 \\
\hline Escore total & & 0,35 & $0,28 / 0,42$ & $<0,001$ & 0,30 & $0,23 / 0,37$ & $<0,001$ \\
\hline
\end{tabular}

Cabe ressaltar que o custo financeiro com os cuidados oferecidos para o paciente depende do local em que a família vive. Famílias que residem em áreas mais desenvolvidas tendem a relatar menos problemas financeiros, uma vez que o acesso aos serviços de saúde e políticas públicas direcionadas para à saúde infantil e ao apoio familiar é diferente quando comparado ao de áreas menos desenvolvidas ${ }^{27}$. Possivelmente a realização do presente estudo nestas áreas teria gerado resultados diferentes, pois a precariedade dos diferentes tipos de recursos pode influenciar a qualidade de vida de seus habitantes.

Em nosso estudo, identificamos que cuidadores que relataram não ter atividade de lazer demonstraram menor percepção de qualidade de vida. Este achado parece ser resultado das dificuldades da dinâmica em que os cuidadores es- tão envolvidos. Crianças cardiopatas necessitam de cuidados contínuos, com múltiplos serviços especiais, tanto de saúde como sociais e familiares. Isso gera uma crescente sobrecarga para seus cuidadores $^{6,28}$. Gerenciar muitas atividades pode limitar o tempo de lazer e os cuidados relacionados à própria saúde. Desta forma, contar com pessoas que possam cooperar nos cuidados com a criança adoecida é uma estratégia efetiva para melhorar a qualidade de vida ${ }^{7,29}$.

Em um estudo com cuidadores de crianças com doenças oncológicas, a impossibilidade de realizar atividades de lazer associou-se à percepção dos cuidadores de serem os únicos capazes de identificar e resolver as necessidades do paciente ${ }^{30}$. Segundo os autores, não ter atividade de lazer também poderia estar relacionado à possível negação de receber ajuda por se sentirem culpa- 
$\operatorname{dos}^{30}$. Nestes casos, os cuidadores acreditam que seria inaceitável abdicar de sua responsabilidade para realizar atividades em benefício próprio ${ }^{31}$.

Identificamos também que cuidadores que fazem tratamento de saúde para doenças como hipertensão arterial sistêmica, diabetes, ansiedade, depressão, doenças osteoarticulares e musculares apresentaram menores escores na escala WHOQOL-BREF. Em conformidade, estudos com cuidadores de crianças com problemas crônicos de saúde apontaram que estes, por sua vez, têm pior condição de saúde ${ }^{32}$. De fato, cuidar exclusivamente de uma criança com doença crônica pode afetar a saúde física e mental dos cuidadores, em consequência à exaustão causada pelas inúmeras tarefas realizadas.

Entre as consequências mais frequentes para os cuidadores estão dores musculares e sintomas depressivos $^{33}$. Também foram identificados fatores de risco cardiovascular em pais ou cuidadores de crianças cardiopatas, como sedentarismo, hipertensão e obesidade ${ }^{34}$. Uma hipótese provável para este achado é que o tempo de cuidado dedicado à criança doente pode diminuir a atenção do cuidador sobre sua própria saúde. Tais problemas de saúde intensificam-se com a gravidade da doença e o aumento de limitações funcionais do paciente ${ }^{35}$.

Em nosso trabalho, a gravidade da cardiopatia não exerceu influência sobre a qualidade de vida dos cuidadores; diferentemente dos resultados do estudo de Arafa et al. ${ }^{6}$, em que a gravidade da cardiopatia associou-se negativamente a todos os domínios da escala de qualidade de vida. Este contraste pode ser explicado pelas diferenças metodológicas entre os dois estudos. Enquanto em nossa pesquisa foi utilizada a classificação de risco cirúrgico e a presença de cianose como critério de gravidade da doença, Arafa et al. ${ }^{6}$ utilizaram um conjunto de informações baseadas no diagnóstico clínico. Além disso, a escala de qualidade de vida utilizada por aqueles autores foi
SF-36, enquanto neste estudo aplicamos a escala WHOQOL-BREF.

Cabe considerar que o delineamento da nossa investigação foi transversal, o que não permite determinar a causalidade entre as variáveis estudadas. Além disso, os pais ou cuidadores foram recrutados em uma amostra de conveniência procedente de famílias de baixo nível socioeconômico e a coleta foi realizada em uma única unidade de atendimento, o que limita o poder de generalização dos resultados.

Por outro lado, os dados foram coletados por uma única pesquisadora, que foi treinada para os procedimentos de campo, e as escalas utilizadas são validadas para representar os eventos estudados. Além disso, em nossa investigação foi utilizado o escore total da escala, que representa de forma abrangente o desfecho estudado, diferentemente da maioria dos trabalhos sobre qualidade de vida, em que os domínios são estudados isoladamente.

Os resultados do presente estudo permitem concluir que receber apoio social, ter melhores condições socioeconômicas, não estar em tratamento de saúde e praticar atividade de lazer estão associados à maior percepção de qualidade de vida. Entre estas variáveis, o apoio social foi o fator mais importante para a explicação do desfecho. $\mathrm{O}$ método utilizado permitiu identificar a associação entre o apoio social e a percepção da qualidade de vida independentemente de outros fatores. Esses aspectos devem ser considerados quando da elaboração de estratégias que visem à melhoria da qualidade de vida desse grupo populacional.

Finalmente, recomendamos estudos longitudinais em pesquisas futuras para identificar em que momento o apoio social seria mais necessário aos cuidadores. Sugerimos também o uso da metodologia qualitativa, que poderia ser a mais adequada para debater esta questão, por permitir maior compreensão da percepção de qualidade de vida e apoio social. 


\section{Colaboradores}

GV Silva participou da redação do artigo, coleta e análise dos dados, elaboração dos resultados e discussão do artigo. DEB Moraes contribuiu na concepção e delineamento do tema de pesquisa, escrita e revisão final do manuscrito. T Konstantyner contribuiu com as análises e interpretação dos dados, elaboração dos resultados, escrita e revisão final do manuscrito. HP Leite contribuiu na concepção e delineamento do tema de pesquisa, orientação para análise dos dados, escrita, revisão e aprovação da versão final do artigo.

\section{Agradecimentos}

Os autores agradecem aos pais por sua contribuição e à equipe da Cardiologia Pediátrica do Hospital Universitário. GV Silva recebeu bolsa de estudos da Coordenação de Aperfeiçoamento de Pessoal de Nível Superior (CAPES).

\section{Referências}

1. Mitchell SC, Korones SB, Berendes HW. Congenital heart disease in 56,109 births. Incidence and natural history. Circulation 1971; 43(3):323-332.

2. Brasil. Ministério da Saúde (MS). Sistema de Informação sobre nascidos vivos (SINASC) [Internet]. [acessado 2018 Out 05]. Disponível em: http://www2.datasus.gov.br/DATASUS/index.php?area $=060702$

3. Letourneau KM, Horne D, Soni RN, McDonald KR, Karlicki FC, Fransoo RR. Advancing Prenatal Detection of Congenital Heart Disease: A Novel Screening Protocol Improves Early Diagnosis of Complex Congenital Heart Disease. J Ultrasound Med 2017; 37(5):1073-1079.

4. Werner H, Latal B, Valsangiacomo Buechel E, Beck I, Landolt MA. The impact of an infant's severe congenital heart disease on the family: a prospective cohort study. Congenit Heart Dis 2014; 9(3):203-210.

5. Kolaitis GA, Meentken MG, Utens EMWJ. Mental Health Problems in Parents of Children with Congenital Heart Disease. Front Pediatr 2017; 5:102.

6. Arafa MA, Zaher SR, El-Dowaty AA, Moneeb DE. Quality of life among parents of children with heart disease. Health Qual Life Outcomes 2008; 6(1):91.

7. Lawoko S, Soares JJF. Social Support among Parents of Children with Congenital Heart Disease, Parents of Children with Other Diseases and Parents of Healthy Children. Scand J Occup Ther 2003; 10(4):177-187.

8. Rempel GR, Neufeld A, Kushner KE. Interactive Use of Genograms and Ecomaps in Family Caregiving Research. J Fam Nurs 2007; 13(4):403-419.

9. Nicolaou C, Papathanassoglou E, Kouta C, Middleton $\mathrm{N}$. Health-related quality of life, social support and social capital of mothers of children with cancer. Eur J Public Health 2015; 25(Supl. 3):112.

10. Leahey M, Wright LM. Enfermeiras e famílias: Um guia para avaliação e intervenção na família. $5^{\mathrm{a}}$ ed. São Paulo: ROCA; 2012.

11. Associação Brasileira de Empresas de Pesquisa [Internet]. Critério de Classificação Econômica Brasil [acessado 2018 Out 05]. Disponível em: http://www.abep. org/criterio-brasil

12. Group TW. The Development of the World Health Organization Quality of Life Assessment Instrument (the WHOQOL). In: Quality of Life Assessment: International Perspectives. Berlin, Heidelberg: Springer Berlin Heidelberg; 1994. p. 41-57.

13. Fleck MPA, Leal OF, Louzada S, Xavier M, Chachamovich E, Vieira G, Santos L, Pinzon V. Desenvolvimento da versão em português do instrumento de avaliação de qualidade de vida da OMS (WHOQOL-100). Rev Bras Psiquiatr 1999; 21(1):19-28.

14. Sherbourne CD, Stewart AL. The MOS social support survey. Soc Sci Med 1991; 32(6):705-714.

15. Griep RH, Chor D, Faerstein E, Werneck GL, Lopes CS. Validade de constructo de escala de apoio social do Medical Outcomes Study adaptada para o português no Estudo Pró-Saúde. Cad Saude Publica 2005; 21(3):703-714. 
16. Anjos KF, Boery RNSO, Pereira R, Pedreira LC, Vilela ABA, Santos VC, Rosa DOS. Association between social support and quality of life of relative caregivers of elderly dependents. Cien Saude Colet 2015; 20(5):1321-1330.

17. Amendola F, Oliveira MAC, Alvarenga MRM. Influência do apoio social na qualidade de vida do cuidador familiar de pessoas com dependência. Rev Esc Enferm USP 2011; 45(4):884-889.

18. Manee F, Ateya Y, Rassafiani M. A Comparison of the Quality of Life of Arab Mothers of Children with and without Chronic Disabilities. Phys Occup Ther Pediatr 2016; 36(3):260-271.

19. Agrawal H, Wright OK, Carberry KE, Sexson Tejtel SK, Mery CM, Molossi S. Family perception of unmet support needs following a diagnosis of congenital coronary anomaly in children: Results of a survey. Congenit Heart Dis 2017; 12(6):721-725.

20. Bratt EL, Järvholm S, Ekman-Joelsson BM, Mattson LÅ, Mellander M. Parent's experiences of counselling and their need for support following a prenatal diagnosis of congenital heart disease - a qualitative study in a Swedish context. BMC Pregnancy Childbirth 2015; 15:171.

21. Fleck MPA, Chachamovich E, Trentini CM. Projeto WHOQOL-OLD: método e resultados de grupos focais no Brasil. Rev Saude Publica 2003; 37(6):793-799.

22. Bruce E, Lilja C, Sundin K. Mothers' lived experiences of support when living with young children with congenital heart defects. J Spec Pediatr Nurs 2014; 19(1):54-67.

23. Neufeld A, Harrison MJ, Hughes K, Stewart M. Nonsupportive interactions in the experience of women family caregivers. Health Soc Care Community 2007; 15(6):530-541.

24. Monteiro M, Matos AP, Coelho R. A adaptação psicológica de mães cujos filhos apresentam Paralisia Cerebral: Revisão da literatura. Rev Port Psicossomática 2002; 4(2):149-178.

25. Inouye K, Pedrazzani ES. Nível de instrução, status socioeconômico e avaliação de algumas dimensões da qualidade de vida de octogenários. Rev Lat Am Enfermagem 2007; 15(esp.):742-747.

26. Medway M, Tong A, Craig JC, Kim S, Mackie F, McTaggart S, Walker A, Wong G. Parental perspectives on the financial impact of caring for a child with CKD. Am J Kidney Dis 2015; 65(3):384-393.

27. Shattuck PT, Parish SL. Financial Burden in Families of Children With Special Health Care Needs: Variability Among States. Pediatrics 2008;122(1):13-18.

28. Jenkins KJ, Gauvreau K, Newburger JW, Spray TL, Moller JH, Iezzoni LI. Consensus-based method for risk adjustment for surgery for congenital heart disease. J Thorac Cardiovasc Surg 2002; 123(1):110-118.

29. Neves ET, Cabral IE, Silveira A. Family network of children with special health needs: implications for Nursing. Rev Lat Am Enfermagem 2013; 21(2):562570 .
30. Beck ARM, Lopes MHBM. Tensão devido ao papel de cuidador entre cuidadores de crianças com câncer. Rev Bras Enferm 2007; 60(5):513-518.

31. Smith GC, Smith MF, Toseland RW. Problems identified by family caregivers in counseling. Gerontologist 1991; 31(1):15-22.

32. Brehaut JC, Kohen DE, Garner RE, Miller AR, Lach LM, Klassen AF, Rosenbaum PL. Health among caregivers of children with health problems: findings from a Canadian population-based study. Am J Public Health 2009; 99(7):1254-1262.

33. Kvarme LG, Albertini-Früh E, Brekke I, Gardsjord R, Halvorsrud L, Liden H. On duty all the time: health and quality of life among immigrant parents caring for a child with complex health needs. J Clin Nurs 2016; 25(3-4):362-371.

34. Borges CF, Busnello FM, Pellanda LC. Identificação de fatores de risco cardiovascular em pais/cuidadores de crianças cardiopatas. Arq Bras Cardiol 2012; 99(4):936-943.

35. Mosher CE, Bakas T, Champion VL. Physical Health, Mental Health, and Life Changes Among Family Caregivers of Patients With Lung Cancer. Oncol Nurs Forum 2013; 40(1):53-61.
Artigo apresentado em 30/06/2018

Aprovado em 15/11/2018

Versão final apresentada em 17/11/2018 
